\title{
Incompatibilities between Y chromosome and autosomes are responsible for male hybrid sterility in crosses between Drosophila virilis and Drosophila texana
}

\author{
KLEA LAMNISSOU†, MICHAEL LOUKAS $\$$ \& ELEFTHERIOS ZOUROS*§ \\ $\dagger$ Department of Biochemistry, Cell and Molecular Biology and Genetics, University of Athens, Athens, $\ddagger$ Department \\ of Agricultural Biology and Biotechnology, Agricultural University of Athens, Athens and \$Department of Biology, \\ University of Crete, Greece and Department of Biology, Dalhousie University, Halifax, Nova Scotia, Canada B3H 4J1
}

\begin{abstract}
Crosses between Drosophila virilis and $D$. texana produce viable and fertile $F_{1}$ males and females. When $F_{1}$ males are backcrossed to either parental species they also produce fertile sons. However, about one-third of $\mathrm{F}_{1}$ males carrying the $D$. texana $\mathrm{Y}$ chromosome are sterile. When fertile $F_{1}$ males with the $D$. texana $\mathrm{Y}$ chromosome are crossed to $D$. virilis, about three quarters of the sons are sterile. We show that these sterilities result from incompatibilities between the $D$. texana $\mathrm{Y}$ chromosome and at least two of the $D$. virilis autosomes. $\mathrm{X} / \mathrm{Y}$ incompatibilities can be excluded in this pair of species, and $\mathrm{X} /$ autosome incompatibilities appear to be either absent or to play a minor role in the sterility of male progeny from backcrosses of $F_{1}$ males to females from either species. It is suggested that Y/autosome incompatibilities may be among the first to appear in the development of postzygotic isolation in Drosophila.
\end{abstract}

Keywords: chromosomal incompatibilities, Drosophila virilis, hybrid sterility.

\section{Introduction}

Genetic studies of hybrid sterility or hybrid inviability are important to biologists for a variety of reasons. From the developmental biologist's point of view, the most interesting question about a genetic factor that causes hybrid sterility or inviability is what the gene's function is under normal conditions and how the gene may help understand the processes of gametogenesis or development to the adult stage (Hutter et al., 1990). From the evolutionary biologist's point of view, the most interesting question is how do separate populations acquire different sets of mutations that are compatible for normal development in homospecific backgrounds but incompatible in heterospecific backgrounds (Coyne, 1992). The fact that homospecific combinations of alleles at a given set of loci can sustain normal development but heterospecific combinations cannot is in itself evidence of the complex epistatic networks that underlie normal development

*Correspondence: Department of Biology, Dalhousie University, Halifax, Nova Scotia, Canada B3H $4 J 1$.
(Davis et al., 1994; Wu \& Palopoli, 1994). The evolution of postzygotic isolation can, therefore, be seen as the development of incompatibilities between genes involved in these networks (Zouros, 1989).

Starting with Dobzhansky (1936), Drosophila has been the favourite organism of genetic studies of postzygotic reproductive isolation (for a recent review see Wu \& Palopoli, 1994). The usual protocol involves bringing genetic material from two different species into the same individual and recording the effect on fertility or viability with the help of phenotypic or molecular markers. When interspecific recombination is allowed in hybrid crosses, marker genes and genes causing hybrid sterility are decoupled and the effect of the marked parts of the genome emerges as a statistical property. This facilitates gaining an insight about the number and distribution of genes involved in postzygotic isolation, but obstructs the detection of synergistic interactions between different genes, except those that are very proximal to the marker genes. For the detection of such interactions one would need to divide the genome into well-defined 
parts that can be transmitted intact in the progeny of hybrid crosses, so that one may ask what conspecific combinations of such parts are indispensable (and what interspecific combinations are incompatible) for fertility or viability. Chromosomes are obvious candidates for such studies provided that interspecific recombination can be avoided. In Drosophila, where recombination does not occur in males, this is possible for pairs of species that produce fertile $F_{1}$ males.

Chromosomal incompatibilities can be classified into four types: between the two sex chromosomes $(\mathrm{X} / \mathrm{Y})$, between the $\mathrm{X}$ chromosome and an autosome (X/A), between the $\mathrm{Y}$ and an autosome (Y/A), and between two autosomes (A/A). Theoretical and experimental attempts to produce an explanation for Haldane's rule (that hybrid sterility or inviability is more likely to occur in the heterogametic sex; Haldane, 1922) have converged on the prominent role of the $\mathrm{X}$ chromosome (Charlesworth et al., 1987; Coyne \& Orr, 1989a; Wu \& Davis, 1993; Zeng \& Singh, 1993; Turelli \& Orr, 1995). Yet, there appears to be no clear consensus as to whether $\mathrm{X} / \mathrm{Y}$, $\mathrm{X} / \mathrm{A}$ or $\mathrm{Y} / \mathrm{A}$ incompatibilities are more prevalent in male hybrid sterility studies (Goulielmos \& Zouros, 1995).

This study takes advantage of male $F_{1}$ fertility in the pair D. virilis and D. texana. In this pair, as in other pairs of this group, both interspecific crosses produce fertile $F_{1}$ hybrids, so that the index of postzygotic isolation (sensu Zouros, 1974) is zero. The two species can, therefore, be considered to be at an early stage of speciation. We ask the following questions. (a) Is it possible that the two species harbour genes that may, in certain combinations, cause male sterility, even if such sterility is not evident in the $F_{1}$ hybrids? (b) If such combinations do exist, will they be detected as incompatibilities between the two sex chromosomes, or between sex chromosomes and autosomes?

\section{Materials and methods}

We have used one strain of $D$. virilis (15010-1000) and one strain of D. texana (15010-1041), provided to us by the National Drosophila Species Resource Center, Bowling Green State University, Bowling Green, Ohio, USA. The two species belong to the virilis phylad, which together with the montana phylad comprise the virilis group (Throckmorton, 1982). Drosophila virilis has six pairs of acrocentric chromosomes (the sex chromosome pair is pair I, and the dot-like chromosome that accounts for less than 1 per cent of the genome is pair VI). In $D$. texana, autosomes II and III have fused into a single automsome. Homologies of chromosomal arms with other Drosophila groups have been established on the basis of conservation of linkage groups (Zouros, 1976; Loukas et al., 1979).

We have used the sperm motility assay (Zouros, 1981) to characterize a male as one with motile or immotile sperm. A detailed description of the method is given in Vigneault \& Zouros (1986). A male is classified as 'motile' if one or more spermatozoa is seen in undulatory movement. Even though sperm motility scored in this way cannot be equated to male fertility (as some males with small amounts of motile sperm are most likely infertile), the assay is a reliable index of defective spermatogenesis and is being used routinely in studies of male hybrid sterility.

The experimental material consisted of two sets of males. One set was scored only for sperm motility, the other was scored for sperm motility and for one or two allozyme loci. In both cases, backcross males were obtained by crossing $F_{1}$ males to females from one or the other species. This assured that no recombinant chromosomes occurred in these males, so that the complete genotype of a backcross male could be identified through the use of one marker for each independently segregating autosome. The fusion of chromosomes II and III in D. texana (which correspond to the $3 \mathrm{R}$ and $3 \mathrm{~L}$ arms of $D$. melanogaster, respectively) reduces the number of autosomes that segregate independently in $F_{1}$ hybrids to three (if chromosome VI is discounted), because a viable gamete from an $\mathrm{F}_{1}$ hybrid must carry either the compound chromosome of $D$. texana or the II and III chromosomes of $D$. virilis. For this reason we use the notation II + III to refer to these chromosomes.

We have used species-specific allozymes as chromosome markers. The $D$. virilis strain was fixed for the slow allele and the D. texana strain was fixed for the fast allele at the leucine aminopeptidase (Lap) locus. This locus maps in the $3 \mathrm{R}$ arm of $D$. melanogaster. We have used this locus to read the chromosomal constitution of backcross males for chromosomes $\mathrm{II}+\mathrm{III}$. The $D$. virilis strain was also found to be fixed for the fast allele and the D. texana to be fixed for the slow allele of the phosphohexose isomerase $(P h i)$ locus, which maps at the $2 \mathrm{R}$ of $D$. melanogaster and, thus, marks chromosome $\mathrm{V}$ of the $D$. virilis group. We could find no enzyme locus that could serve as a marker of the fourth chromosome, which corresponds to $2 \mathrm{~L}$ of $D$. melanogaster. Three loci known to map at this arm (alcohol dehydrogenase, malate dehydrogenase and $\alpha$-glycerophosphate 
dehydrogenase) were fixed for the same allele in both strains. The methods used for allozyme scoring were as described in Loukas et al. (1979).

\section{Results}

Table 1 gives the number of males with motile and immotile sperm in the two pure species, the two types of $F_{1}$ hybrids and the progeny from the four possible types of backcrosses of $F_{1}$ males. In one $F_{1}$ and three backcross classes the frequency of males with immotile sperm is low and statistically the same as that of pure species (chi-squared test of homogeneity for all six classes $=3.42$ on 5 d.f., $P>0.5$ ), giving an overall rate of baseline sterility of 3.3 per cent. One-third of $F_{1}$ males from the ' $D$. virilis female $\times D$. texana male' cross are sterile and threequarters of the sons from these $F_{1}$ males to $D$. virilis are also sterile.

A simple $\mathrm{X} / \mathrm{Y}$ interaction cannot explain the results. The $\mathrm{X}^{t} \mathrm{Y}^{\mathrm{v}}$ combination is clearly compatible with male fertility, as evidenced from classes 3 and 5. Because both classes with elevated frequencies of males with immotile sperm have the $\mathrm{X}^{\mathrm{v}} \mathrm{Y}^{\mathrm{t}}$ combination, one may hypothesize that this sex chromosome combination is incompatible for male fertility. But this cannot explain why 66 per cent of $F_{1}$ males carrying this combination are fertile, nor why this percentage drops to 25 among backcross male with the same combination of sex chromosomes. An interaction between the $\mathrm{X}$ chromosome and the autosomes could not, also, explain the results, because classes 6 and 8 are the same with regard to $X$ and autosomes (they are both of the type $X^{v}$ $\mathrm{A}^{v} \mathrm{~A}^{\mathrm{t} / \mathrm{v}}$, yet they are very different in their percentage of males with immotile sperm.

To explain these observations we produced a new set of males from the last backcross of Table 1 . Subsets of these males were scored for sperm motility and for one or two enzyme loci, as shown in Table 2. No male that was homozygous for the $D$. virilis homologue of autosome $\mathrm{V}$ was fertile (out of 39 observed). This complete sterility of males of the chromosomal type $X^{v} Y^{t} V^{v} V^{v}$ establishes a $Y / A$ incompatibility, one between the $\mathrm{Y}$ chromosome of $D$. texana and the fifth chromosome of $D$. virilis (incomptibility of the type $\mathrm{Y}^{i} / \mathrm{A}^{j j}$, where $i, j$ stand for different species).

There is also a much higher number of males with immotile sperm among homozygotes for the II + III autosomes than among heterozygotes. The distribution of motile to immotile males with regard to these chromosomes is not different between the second and third part of Table 2 . Thus, the overall rate of immotility among II + III heterozygotes is $0.615(N=39, \quad \mathrm{SE}=0.078)$ and among II + III homozygotes $0.907(N=43, \mathrm{SE}=0.044)$. From the comparison of confidence intervals, the probability that the two rates are equal is $P=0.016$. These rates include the sterility that is caused because of homozygosity for the $D$. virilis chromosome V. The last two entries of Table 2 allow one to obtain a crude estimate of the effect on sperm motility of chromosomes II + III, when the effect of chromosome V is

Table 1 Distribution of male Drosophila with motile and immotile sperm in eight types of crosses

\begin{tabular}{|c|c|c|c|c|}
\hline & Genotype & $\mathbf{M}$ & I & Frequency I (SE) \\
\hline $\begin{array}{l}\text { Pure species } \\
\text { tex } \times \text { tex } \\
\text { vir } \times \text { vir }\end{array}$ & $\begin{array}{l}X^{t} Y^{t} A^{t} A^{t} \\
X^{v} Y^{v} A^{v} A^{v}\end{array}$ & $\begin{array}{l}80 \\
73\end{array}$ & $\begin{array}{l}2 \\
1\end{array}$ & $\begin{array}{l}0.024(0.017) \\
0.013(0.013)\end{array}$ \\
\hline $\begin{array}{l}F_{1} \\
\text { tex } \times \text { vir } \\
v i r \times t e x\end{array}$ & $\begin{array}{l}X^{t} Y^{v} A^{t} A^{v} \\
X^{v} Y^{t} A^{t} A^{v}\end{array}$ & $\begin{array}{l}190 \\
118\end{array}$ & $\begin{array}{r}7 \\
60\end{array}$ & $\begin{array}{l}0.035(0.013) \\
0.337(0.035)\end{array}$ \\
\hline $\begin{array}{l}\text { Backcross } \\
\text { tex } \times(\text { tex } \times \text { vir }) \\
\text { vir } \times(\text { tex } \times \text { vir }) \\
\text { tex } \times(\text { vir } \times \text { tex }) \\
\text { vir } \times(\text { vir } \times \text { tex })\end{array}$ & $\begin{array}{l}X^{t} Y^{v} A^{t} A^{t / v} \\
X^{v} Y^{v} A^{v} A^{t / v} \\
X^{t} Y^{t} A^{t} A^{t / v} \\
X^{v} Y^{t} A^{v} A^{t / v}\end{array}$ & $\begin{array}{r}192 \\
164 \\
100 \\
53\end{array}$ & $\begin{array}{r}10 \\
5 \\
2 \\
161\end{array}$ & $\begin{array}{l}0.049(0.015) \\
0.029(0.013) \\
0.020(0.014) \\
0.752(0.030)\end{array}$ \\
\hline
\end{tabular}

Female parent is shown first.

tex, D. texana; vir, D. virilis; $\mathrm{M}$, number of males with motile sperm; I, number of males with immotile sperm; SE, standard error; A, X, Y stand for autosomes, X chromosome and $\mathrm{Y}$ chromosome, respectively. 
Table 2 The effect of chromosomes V and II + III on sperm motility of male Drosophila from the backcross vir $\times($ tex $\times$ vir $)$

\begin{tabular}{lccc}
\hline & $\mathrm{M}$ & $\mathrm{I}$ & Frequency I (SE) \\
\hline $\begin{array}{l}\text { Chromosome V } \\
\text { virilis/virilis }\end{array}$ & 0 & 25 & 1 \\
virilis/texana & 14 & 17 & $0.548(0.089)$ \\
& $P=4.6 \times 10^{-6}$ & \\
Chromosomes II + III & & & \\
virilis/virilis & 2 & 22 & $0.917(0.056)$ \\
virilis/texana & 10 & 14 & $0.583(0.101)$ \\
& $P=0.009$ & \\
Chromosomes V and II + III & & & \\
virilis/virilis, virilis/virilis & 0 & 7 & 1 \\
virilis/virilis, virilis/texana & 0 & 7 & 1 \\
virilis/texana, virilis/virilis & 2 & 10 & $0.833(0.108)$ \\
virilis/texana, virilis/texana & 5 & 3 & $0.375(0.171)$ \\
& $\chi^{2}=13.11$, d.f. $=3, P<0.005$ \\
\hline
\end{tabular}

Probabilities $(P)$ for homogeneous distribution of males with motile $(\mathrm{M})$ and immotile (I) sperm were calculated from Fisher's exact test (first two parts of table) or from the contingency chi-squared test (third part of table).

removed. The immotility rate is 0.375 for II + III heterozygotes and 0.833 for II + III homozygotes. To substantiate further the effect of chromosomes II + III, 19 males from the same backcross that had motile sperm (and could, therefore, be safely assumed to be heterozygous for chromosome V) were scored for Lap. Of these, 15 were heterozygous $\left(\mathrm{II}+\mathrm{III}^{\mathrm{vt}}\right.$ ) and four were homozygous (II + III ${ }^{\mathrm{vv}}$ ) (chi-squared for 1:1 segreation $=6.37$ on 1 d.f., $P<0.02$ ). We conclude that there is, also, an incompatibility between the $\mathrm{Y}$ chromosome and chromosomes II $+\mathrm{III}^{\mathrm{v}}$, but unlike that of chromosome $\mathrm{V}$, this is not a complete incompatibility in the sense that not all $\mathrm{Y}^{\mathrm{t}} \mathrm{II}+\mathrm{III}^{\mathrm{w} v}$ males have immotile sperm.

To explain the incompleteness of the Y/II + III incompatability we may hypothesize that the fourth chromosome (for which we have no marker) must also be in the heterozygous condition for a male to have motile sperm. This amounts to requiring that in $\mathrm{X}^{\mathrm{v}} \mathrm{Y}^{\mathrm{t}}$ hybrids all major autosomes be in the heterozygous state for sperm motility. If this were true, we would expect half of $\mathrm{V}^{\mathrm{vt}} \mathrm{II}+\mathrm{III}^{\mathrm{vt}}$ males to have immotile sperm (which cannot be ruled out from the data of Table 2) and all II + III ${ }^{\mathrm{vv}}$ to be sterile (which can be ruled out from the data of Table 2). We are, therefore, left with the less satisfactory hypothesis that the interaction of chromosome II + III (and of chromosome IV, if any) with the Y chromosome has incomplete penetrance.

The results of Table 2 are summarized in Fig. 1 which is a model of chromosomal control of $D$. virilis $/ D$. texana hybrid fertility in males with the $\mathrm{X}^{\mathrm{v}} \mathrm{Y}^{\mathrm{t}}$ combination. The presence of the $\mathrm{Y}^{\mathrm{t}}$ chromosome in these males necessitates the presence of a conspecific gene (or genes) in the fifth chromosome. When this condition is fulfilled, fertility will depend on the presence of a conspecific gene (or genes) in chromosome arm II or III (or both). If both conditions are fulfilled, then the current estimate of the probability that the male will have motile sperm is 0.625 ; if the first condition is fulfilled but the second is not, the estimate of this probability is 0.167 .

Because this hypothesis was deduced from the data of Table 2, it is of interest to ask whether it is compatible with the data of Table 1 . There are two classes $(4$ and 8$)$ with the $X^{\mathrm{v}} Y^{t}$ combination. All $F_{1}$ males with the $\mathrm{Y}$ chromosome of $D$. texana (class 4 ) are heterozygous for the V and the II+ III chromosomes, so the expected number of males with immotile sperm is $0.375 \times 178$ or 66.75 as compared to 60 observed (chi-squared from test of fit $=1.09$ on 1 d.f., $P>0.5$ ). Among males of class 8 , one-half will be homozygous for chromosome $\mathrm{V}$ and, therefore, will have immotile sperm. Of the other half, one-half will also be homozygous for chromosomes II + III and will have immotile sperm at a rate of 0.833 . The other half will be heterozygous and will have immotile sperm at a rate of 0.375 . Thus the overall expected frequency of males with immotile sperm is 0.803 , giving an expected number of 171.6 among 
214 examined, as compared to 161 observed (chisquared $=3.3,0.1>P>0.05)$. We conclude that the data of Table 1 are compatible with the model of Fig. 1.

\section{Discussion}

Our analysis has established the following points.

(a) At least two of the four major autosomes contain one or more factors that interact adversely with the foreign $\mathrm{Y}$ chromosome to cause spermatogenetic abnormalities (Y/autosome incompatibilities). One of these incompatibilities has complete and the other has incomplete penetrance.

(b) Y/autosome incompatibilities are asymmetrical with regard to the $Y$ chromosome: they occur when the $\mathrm{Y}$ chromosome is of $D$. texana but not when it is of $D$. virilis origin.

(c) Y/autosome incompatibilities are 'recessive' with regard to the autosome: sperm immotility occurs only if the autosome is in the homozygous condition.

In their analysis of hybrid sterility in the $D$. virilis group, Orr \& Coyne (1989) also scored sperm motility in the $F_{1}$ from $D$. virilis and $D$. texana. They found that the rate of immotility was 0.05 for males with the $D$. virilis $\mathrm{Y}$ chromosome and 0.34 for males

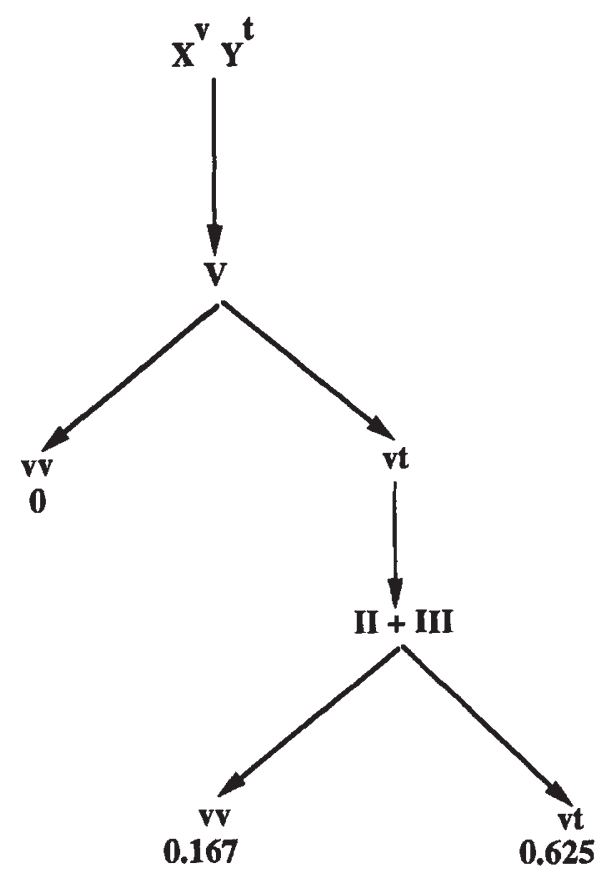

Fig. 1 A model for sperm motility in male Drosophila with the $\mathrm{X}$ chromosome of $D$. virilis and the $\mathrm{Y}$ chromosome of D. texana. X, Y, V and II + III stand for the X, the Y, the fifth and the 'second plus third' chromosomes, respectively; $\mathrm{v}$ and $\mathrm{t}$ stand for $D$. virilis or $D$. texana origins; numbers give the fraction of males with the motile sperm. with the $D$. texana $\mathrm{Y}$ chromosome, a result remarkably similar to ours. Orr \& Coyne also recorded sperm immotility in backcross males, but as these were produced from $F_{1}$ females (and therefore contained recombinant $\mathrm{X}$ chromosomes and autosomes), their results are not comparable to ours.

The results of this study are very similar to those obtained in the sibling species $D$. arizonae and $D$. mojavensis of the $D$. repleta group. When the $\mathrm{F}_{1}$ with the $\mathrm{Y}$ chromosome of $D$. arizonae is crossed to $D$. mojavensis, all backcross males that are homozygous for the fourth chromosome are unconditionally sterile $\left(\mathrm{Y}^{\mathrm{a}} / \mathrm{IV}^{\mathrm{mm}}\right.$ incompatability). Whether the heterozygotes for the fourth chromosome will be sterile or fertile depends on the origin of the $D$. arizonae strain. In some strains, these males are always fertile; in others they are fertile if they are also heterozygous for the third chromosome, otherwise they are always sterile ( $\mathrm{Y}^{\mathrm{a}} / \mathrm{III}^{\mathrm{mm}}$ incompatibility) (Zouros et al., 1988). Thus, in the D. mojavensis/D. arizonae species pair chromosome IV appears to play the role that chromosome $\mathrm{V}$ plays in $D$. virilis/ D. texana and chromosome III to play the role of II + III, except that males $\mathrm{Y}^{\mathrm{a}}$ IV $^{\mathrm{am}} \mathrm{III}^{\mathrm{am}}$ are always fertile whereas $\mathrm{Y}^{t} \mathrm{~V}^{\mathrm{tv}} \mathrm{II}+\mathrm{III}^{\mathrm{tv}}$ may not be. Chromosomes IV and III of the D. repleta group are homologous to chromosomes III and IV of the $D$. virilis group, respectively (Zouros, 1976). Thus, there appears to be no correspondence between homologies of autosomes and effects on hybrid sperm motility among Drosophila species groups. This in turn suggests that a considerable number of autosomal genes of Drosophila may interact with the $\mathrm{Y}$ chromosome during spermatogenesis.

Of the four possible types of chromosomal incompatibilities, X/A appear to be most and A/A least prevalent in male hybrid sterility studies. Indeed, there is only one known case of A/A incompatibility causing male hybrid sterility (Schafer, 1978). This, however, may be so because incompatibilities involving sex chromosomes are more easily detected than A/A incompatibilities. X/A incompatibilities were found in all Drosophila groups studied [the melanogaster (Johnson et al., 1992; Zeng \& Singh, 1993), the obscura (Orr, 1989); the repleta (Zouros et al., 1988) and the virilis (Heikkinen \& Lumme, 1991)]. Even though $\mathrm{X} / \mathrm{Y}$ incompatibilities are thought to be important, they are in fact difficult to establish (or eliminate) because in most studies they cannot be separated from incompatibilities of the type $\mathrm{X}^{i} / \mathrm{A}^{i j}$. Incompatibilities of the latter type may not be rare. When a specifically designed protocol of crosses was applied to the $D$. mojavensis $/ D$. arizonae pair, it revealed that all major autosomes were involved in 
incompatibilities of this kind (Zouros et al., 1988). $\mathrm{X} / \mathrm{Y}$ incompatibilities can be excluded for the pair examined here: $F_{1}$ or backcross hybrids with the $\mathrm{X}^{\mathrm{t}} \mathrm{Y}^{\mathrm{v}}$ combination are fertile and the sterility found in hybrids carrying the reciprocal combination is shown to result from interactions between the $\mathrm{Y}$ of $D$. texana and $D$. virilis autosomes. There are two other well established cases of Y/A incompatibilities: in D. hydei/D. neohydei (Schafer, 1978) and in $D$. arizonae/D. mojavensis (Vigneault \& Zouros, 1986). Indications for $\mathrm{Y} / \mathrm{autosome}$ incompatibilities also exist for $D$. macrospina macrospina/D. macrospina limpiensis (Mainland, 1941) and two transitional semispecies of $D$. paulistorum (Ehrman, 1963). We note that in all these cases the species pairs involved produce fertile $F_{1}$ femles from both reciprocal hybrid crosses and fertile $F_{1}$ males from at least one cross. Thus, Y/A incompatibilities seem to appear very early in the development of post-zygotic isolation.

Our experimental protocol does not allow us to ask if the incompatibilities we have seen are caused by one or more loci. In the case of the II + III incompatibility we cannot even know if the effect results from loci on one or the other (or both) chromosomal arms. Rather than asking the question 'How many genes are there that may cause male hybrid sterility?' we have asked the question 'Can we demonstrate the existence of interchromosomal incompatibilities causing hybrid sterility?' Both questions are important for an understanding of the development of hybrid sterility. The empirical evidence suggests that the number of hybrid sterility genes can be large, even in very closely related species (Wu \& Palopoli, 1994), which means that a meaningful answer to the first question cannot be obtained without the availability of a large number of genetic markers. Our approach to the second question is also limited, because it cannot detect intrachromosomal interactions. We may suspect from the work of Davis et al. (1994) on the distal end of the $\mathrm{X}$ chromosome of $D$. sechellia/D.simulans that epistatic networks among closely linked loci are common. However, our approach is useful if the objective is to assess what type of interchromosomal incompatibilities are more likely to appear at the very early stages of the development of postzygotic isolation. At least for the pair of species and the backcross males examined here, Y/A incompatibilities appear to be more important than $\mathrm{X} / \mathrm{Y}$ or $\mathrm{X} / \mathrm{A}$ incompatibilities.

Because fertile individuals are found in large numbers in male and female $F_{1}$ progeny from both reciprocal crosses of $D$. virilis and $D$. texana, this pair's index of postzygotic reproductive isolation (sensu Zouros, 1974) is zero (e.g. Coyne \& Orr, 1989b). We have demonstrated here [as have Orr \& Coyne (1989) and Heikkinen \& Lumme (1991) in the same Drosophila species group] that $F_{1}$ hybrid fertility does not imply that two species have not accumulated mutations which, in combination, are incompatible for male fertility. The fertility or sterility of $F_{1}$ hybrids can be used as a crude indication of the true level of postzygotic isolation in comparative studies (Zouros, 1974; Coyne \& Orr, $1989 \mathrm{~b}$ ), but the qualitative statement that two species may have acquired ethological isolation in the absence of postreproductive isolation may be misleading when based solely on $\mathrm{F}_{1}$ performance. In Drosophila there is at present only one case in which this appears to be true, the Zimbabwe strain of $D$. melanogaster which shows strong ethological isolation from other conspecific strains (Wu et al., 1995). Given the current division of opinion about the potential of postreproductive isolation to promote premating isolation (Noor, 1995), it is important that the former type of isolation be explored beyond the $\mathrm{F}_{1}$ stage.

\section{Acknowledgements}

This research was supported by a Natural Sciences and Engineering Research Council (Canada) grant to E. Z.

\section{References}

CHARLESWORTH, B., COYNE, J. A. AND BARTON, N. H. 1987. The relative rates of evolution of sex chromosomes and autosomes. Am. Nat., 130, 113-146.

COYNE, J. A. 1992. Genetics and speciation. Nature, 355, $511-515$

COYNE, J. A. AND ORR, H. A. 1989a. Two rules of speciation. In: Otte, D. and Endler, J. (eds) Speciation and its Consequences, pp. 180-207. Sinauer Associates, Sunderland, MA.

COYNE, J. A. AND ORR, H. A. 1989b. Patterns of speciation in Drosophila. Evolution, 43, 362-381.

DAVIS, A. W., NOONBURG, E. G. AND WU, C.-I 1994. Evidence for complex interactions between conspecific chromosomes underlying hybrid female sterility in the Drosophila simulans clade. Genetics, 137, 191-199.

DOBZHANSKY, T. 1936. Studies of hybrid sterility. II. Localization of sterility factors in Drosophila pseudoobscura hybrids. Genetics, 21, 113-135.

EHRMAN, L. 1963. Apparent cytoplasmic sterility in Drosophila paulistorum. Proc. Natl. Acad. Sci. U.S.A., 49, 155-158.

GOULIELMOS, G. AND zOUROS, E. 1995. Incompatibility analysis of male hybrid sterility in two Drosophila 
species: lack of evidence for maternal, cytoplasmic, or transposable element effects. Am. Nat., 145, 1006-1014. HALDANE, J. B. s. 1922. Sex-ratio and unisexual sterility in hybrid animals. J. Genet., 12, 101-109.

HEIKKINEN, E. AND LUMME, J. 1991. Sterility of male and female hybrids of Drosophila virilis and Drosophila lummei. Heredity, 67, 1-11.

HUTtER, P., ROOTE, J. AND ASHBURNeR, M. 1990. A genetic basis for the inviability of hybrids between sibling species of Drosophila. Genetics, 124, 909-920.

JOHNSON, N. A., PEREZ, D. E., CABOT, E. L., HOLLOCHER, H. AND WU, C.-I 1992. A test of reciprocal X-Y interactions as a cause of hybrid sterility in Drosophila. Nature, 358, 751-753.

LOUKAS, M., KRIMBAS, C. B., MAVRAGANI-TSIPIDOU, P. AND KASTRITSIs, C. D. 1979. Genetics of Drosophila subobscura populations. VIII. Allozyme loci and their chromosome maps. J. Hered., 70, 17-26.

MAINLAND, G. B. 1941. Studies in speciation. III. The Drosophila macrospina group. Genetics, 26, s160.

NOOR, M. A. 1995. Speciation driven by natural selection in Drosophila. Nature, 375, 674-675.

ORR, H. A. 1989. Genetics of sterility in hybrids between two subspecies of Drosophila. Evolution, 43, 180-189.

ORR, H. A. AND COYNE, J. A. 1989. The genetics of postzygotic isolation in the Drosophila virilis group. Genetics, 121, 527-537.

SCHAFER, U. 1978. Sterility in Drosophila hydei $\times$ Drosophila neohydei hybrids. Genetica, 49, 205-214.

THROCKMORTON, L. H. 1982. The virilis group. In: Ashburner, M., Carson, H. L. and Thompson, J. N., Jr. (eds) The Genetics and Biology of Drosophila, vol. 3b, pp. 227-296. Academic Press, London.

TURRELI, M. AND ORR, H. A. 1995. The dominance theory of Haldane's rule. Genetics, 140, 389--402.

VIGNEAULT, G. AND zouros, E. 1986. The genetics of asymmetrical male sterility in Drosophila mojavensis and Drosophila arizonensis hybrids: interactions between the Y chromosomes and autosomes. Evolution, 40, $1160-1170$.

wU, C.-I AND DAvIS, A. w. 1993. Evolution of postmating reproductive isolation: the composite nature of Haldane's rule and its genetic bases. Am. Nat., 142, $187-212$.

WU, C.-I AND PALOPOLI, M. F. 1994. Genetics of postmating reproductive isolation in animals. Ann. Rev. Genet., 28, 283-308.

WU, C.-I, HOLLOCHER, H., BEGUN, D. J., AQUADRO, C. F. AND XU, Y. 1995. Sexual isolation in Drosophila melanogaster: a possible case of incipient speciation. Proc. Natl. Acad. Sci. U.S.A., 92, 2519-2523.

ZENG, L.-W. AND SINGH, R. S. 1993. The genetic basis of Haldane's rule and the nature of asymmetric hybrid male sterility between Drosophila simulans, D. mauritiana and D. sechellia. Genetics, 134, 251-260.

zouros, E. 1974. Genic differentiation associated with the early stages of speciation in the mulleri subgroup of Drosophila. Evolution, 27, 601-621.

zOUROS, E. 1976. The distribution of enzyme and inversion polymorphism over the genome of Drosophila: evidence against balancing selection. Genetics, 83, 169-179.

ZOUROS, E. 1981. An autosoma-Y chromosome combination that causes sterility in $D$. mojavensis $\times D$. arizonensis hybrids. Dros. Inf. Serv., 56, 167-168.

zouros, E. 1989. Advances in the genetics of reproductive isolation in Drosophila. Genome, 31, 211-220.

ZOUROS, E., LOFDAHL, K. AND MARTIN, P. A. 1988. Male hybrid sterility in Drosophila: interactions between autosomes and sex chromosomes in crosses of D. mojavensis and D. arizonensis. Evolution, 42, 1321-1331. 